\title{
ALIADOS EXTRAÑOS: LA INFERENCIA A LA MEJOR EXPLICACIÓN Y EL ESTÁNDAR DE PRUEBA PENAL*
}

\author{
Larry LAUDAN**
}

\begin{abstract}
Resumen:
En este artículo, el autor aborda la cuestión fundamental de si el modelo de la Inferencia a la Mejor Explicación (IME) es adecuado o no para fungir como sustituto del Estándar de Prueba ( $\mathrm{E}$ de $\mathrm{P}$ ) actual que rige en materia penal en los Estados Unidos de América (prueba más allá de toda duda razonable). Luego de una breve exposición del esquema general de la IME propuesto en los terrenos de la epistemología y la filosofia de la ciencia, y luego de concluir que se trata de un modelo deficiente para explicar la actividad consistente en la aceptación y rechazo de teorías científicas, el autor procede al análisis específico de su aplicación en el campo de la decisión (de un juez o jurado) relativa a la culpabilidad o inocencia del penalmente inculpado.
\end{abstract}

Abstract:

In this short essay the author deals with the fundamental question of whether the Inference to the Best Explanation (IBE) model has sufficient grounds to be considered as a substitute of the current criminal standard of proof (proof Beyond All Reasonable Doubt). After giving an overview of the IBE model as proposed in more general fields such as epistemology and the philosophy of science, and after concluding that the IBE has failed as a model of the acceptance and rejection of scientific theories, the author analysis its plausibility when applied as a decision rule for inferring if the accused is guilty or not.

* Traducción de Edgar R. Aguilera, estudiante del doctorado en derecho por investigación en el Instituto de Investigaciones Jurídicas de la UNAM, y asistente del proyecto CONACYT "La epistemología del derecho penal mexicano" coordinado por Larry Laudan.

** Investigador en el Instituto de Investigaciones Filosóficas de la UNAM. Estoy muy agradecido con Amalia Amaya, Jordi Ferrer y Ron Allen por sus críticas a una versión preliminar de este ensayo. 
Uno de los problemas en decir que el razonamiento abductivo es una inferencia a la mejor explicación es que puede ser que no contemos con un criterio establecido para determinar cuál es la mejor explicación.

David SCHUM ${ }^{1}$

SUMARIO: I. Introducción. II. Elementos generales de la inferencia a la mejor explicación. III. Estándares de prueba juridicos e IME. IV. Conclusiones.

\section{INTRODUCCIÓN}

Hace casi medio siglo, ${ }^{2}$ desde que Gil Harman acuñara el término de Inferencia a la Mejor Explicación (en adelante IME), numerosos epistemólogos y filósofos de la ciencia han venido explorando su idea de que la regla de decisión para la aceptación de hipótesis y teorías debe basarse en las virtudes explicativas de los diversos candidatos en consideración. En pocas palabras, Harman sugería que si podemos determinar cuál de las hipótesis conocidas que explican los hechos en cuestión, hace el mejor trabajo al explicarlos, entonces esa es la hipótesis que debe aceptarse, derrotablemente, como verdadera.

Recientemente, varios académicos del derecho, incluyendo a Ron Allen y a Michael Pardo, han sugerido que el modelo de la IME puede proveer de un instrumento intelectual crucial para roer el persistentemente duro hueso

1 Schum, David A., "Species of Abductive Reasoning in Fact Investigation in Law", Cardozo Law Review, núm. 22, 2001, pp.1645-1659.

2 El Locus classicus es: Harman, G., "The inference to the best explanation", 74 Philosophical Review 88, 1965. Puede decirse que el modelo de Harman es una especificación particular de lo que Peirce anteriormente habia llamado inferencia abductiva. De este modo, la inferencia a la mejor explicación sufre de todas las debilidades epistemológicas generales de la inferencia abductiva, además de aquellas que tienen que ver con el requisito que Harman agrega en el sentido de exigir una relación de explicatividad (en oposición a una relación de implicación) entre las premisas y la conclusión de una inferencia a la mejor explicación. 
de definir, en forma robusta, los estándares de prueba jurídicos. ${ }^{3}$ Fácilmente puede apreciarse el atractivo inicial de esta estrategia. Existe un acuerdo general en el sentido de que el estándar penal actual, prueba más allá de toda duda razonable, es confuso, se encuentra deficientemente definido, y frecuentemente es ininteligible para los jurados (nótese el hecho de que es común que los jurados soliciten a los jueces que clarifiquen la noción). Incluso el estándar civil, prueba por una preponderancia de la evidencia, el cual generalmente es entendido como que sea más probable el hecho que su negación, parece estar tristemente ligado a una teoria de estimaciones probabilisticas subjetivas que los jurados no son capaces de implementar con algún grado de confiabilidad en la práctica. En estas circunstancias, dificilmente sorprende que los académicos del derecho exploren discusiones más generales en el terreno de la epistemología buscando ideas prometedoras en las cuales fundar una teoría general de la prueba jurídica, así como nuevas explicaciones de los estándares de prueba penal y civil en particular.

He dedicado gran parte de mi vida académica a argumentar que la IME es un mal modelo para el conocimiento científico. ${ }^{4}$ He mostrado que el modelo de la IME rutinariamente autoriza la aceptación de teorías e hipótesis científicas cuya falsedad ha sido revelada en repetidas ocasiones por la investigación empírica posterior. He sostenido que la IME es un criterio demasiado permisivo para usarse como la base sobre la cual tomar decisiones acerca de qué teorias científicas hay que creer, debido ni más ni menos, a que irremediablemente se ve constreñida a las hipótesis que de antemano nos hemos arreglado para inventar (de entre las cuales, el modelo nos insta a escoger "la mejor"). Una y otra vez, teorias que indiscutiblemente eran las mejores explica-

3 Allen, Ron y Pardo, Michael, "The Problematic Value of Mathematical Models of Evidence", Journal of Legal Studies, forthcoming, 2007, p. 41.

4 Véase Laudan, Larry, "A Confutation of Convergent Realism", 48 Philosophy of Science, núm. 19, 1981; y Beyond Positivism and Relativism, Boulder, Westview Press, 1996. 
ciones científicas de su tiempo (la astronomía ptolomeica, la mecánica newtoniana, el atomismo químico clásico, la fisiología galénica, la teoría corpuscular de la luz, teorías del éter electromagnético), subsecuentemente se mostró que eran falsas. En concreto, la IME como estrategia para la evaluación de creencias en las ciencias naturales, no sólo es en principio falible (algo con lo que podríamos vivir), sino que frecuentemente e incluso, sistemáticamente, nos conduce a aceptar creencias acerca del mundo natural respecto de las cuales subsecuentes aplicaciones del propio modelo de la IME muestran que son falsas. Por tanto, el modelo de la IME es contundentemente socavado por la denominada "inducción escéptica o pesimista" de la historia de la ciencia.

Sin embargo, y esto es crucial, el fracaso de la IME para calificar como una adecuada epistemología de la ciencia no implica que fracasará como una epistemología del derecho. ${ }^{5}$ El mal desempeño de la IME en las ciencias se deriva del hecho de que los científicos constantemente someten a una revisión a gran escala las historias acerca de aquello de lo que está hecho el universo. La IME no funciona como una epistemología de la ciencia porque no logra llegar a buenos términos con el hecho ineluctable de que en forma periódica los científicos revisan sus creencias más fundamentales y arraigadas. ${ }^{6}$ Afortunadamente, para los defensores de la IME esto no pasa en el derecho. Sin duda, ocasionalmente descubrimos que ciertos jurados han cometido errores en juicios particulares; sin embargo, ningún estudioso de las decisiones judiciales cree que los veredictos sean falsos la mayoria de las veces. En suma, no hay contraparte jurídica de este estremecedor descubrimiento sobre la ciencia, de

5 Aunque, para sonar algo cínico, uno se pregunta por qué los académicos del derecho habrian de sentirse inclinados a tomar prestado, de la filosofia de la ciencia, un modelo para la evaluación de hipótesis que se ha desempeñado tan mal como una teoría de la inferencia científica.

6 Con cada revolución científica importante, somos testigos de un cambio en la ontologia subyacente, el cual vicia parcialmente las afirmaciones precedentes acerca de qué es lo que existe y, en este sentido, repudia las "mejores" explicaciones anteriores sobre aquello que hay en el mundo (siempre y cuando éstas hayan sido formuladas en los términos de la ontología subyacente). 
que nuestros predecesores aceptaron, en forma regular, teorias falsas (las cuales eran las mejores explicaciones disponibles en su momento) acerca de los fenómenos que trataban de entender.

Dicho lo anterior, no obstante, pienso que hay razones poderosas, específicamente relativas al derecho (en particular, al derecho penal), para dudar que efectivamente la IME puede arrojar luces a las cuestiones sobre la naturaleza de los estándares de prueba jurídicos, lo cual parece ser la principal motivación del creciente coqueteo de los teóricos de la evidencia y los epistemólogos del derecho con esta estrategia epistemológica en particular. En este corto ensayo quiero analizar algunas de esas dudas. Específicamente, deseo concentrarme en la pregunta de si la IME es un candidato plausible para cimentar nuestras reflexiones acerca del estándar de prueba en el derecho, especialmente, del estándar de prueba en materia penal.

No son pocos los académicos del derecho que piensan que la IME puede ser un modelo prometedor. Recientemente Ron Allen y Michael Pardo han sostenido que "la mejor forma de explicar la prueba judicial consiste en considerar que aquella involucra la inferencia a la mejor explicación de principio a fin". ${ }^{7}$ Esta referencia a principios y finales alude a la tesis de Allen y Pardo de que la IME opera no sólo a nivel micro (gobernando las decisiones acerca de la confiabilidad que se otorga a ciertas piezas de la evidencia consideradas en lo individual), sino igualmente a nivel macro (al nivel de la decisión de culpabilidad o inocencia). John Josephson en su amplio estudio "On the Proof. Dynamics of Inference to the Best Explanation", en forma similar, ha argumentado en favor de que la IME nos permite generar "una definición para el estándar judicial de prueba "más

7 Allen, Ron y Pardo, Michael, "The Problematic Value of Mathematical Models of Evidence", Journal of Legal Studies, forthcoming, 2007, p. 41 (las cursivas son mías). 
allá de toda duda razonable»".8 Paul Thagard ha hecho aseveraciones parecidas, la cuales se mencionaran más adelante.

La pregunta central del ensayo es si la IME esclarece o no nuestro concepto del estándar de prueba penal, o incluso, si en principio se encuentra siquiera en condiciones de poder hacerlo. Aunque tendré mucho menos que decir sobre la IME como estándar civil que como estándar penal, brevemente ofreceré razones, para ser escéptico, acerca de su utilidad como un mecanismo que captura la noción del estándar de prueba en el derecho civil. Para anticipar en un par de frases, las conclusiones a las que eventualmente seremos dirigidos, sostendré que la IME es demasiado débil para fungir como el estándar penal, y demasiado fuerte para fungir como el civil (o obstante, si alguno todavía ansía encontrar un papel para la IME en el derecho, puede decirse que tal vez capturaría el significado del estándar de "evidencia clara y convincente", o quizá pueda modelar las micro-decisiones acerca del peso adecuado que debe atribuirse a piezas o elementos específicos de la evidencia o los testimonios).

\section{ElEMENTOS GENERALES DE LA INFERENCIA \\ A LA MEJOR EXPLICACIÓN}

Antes que otra cosa, la IME pretende ser una regla de desprendimiento. Este modelo especifica una serie de premisas que, de ser satisfechas en el caso concreto, justificarían que infiriéramos la verdad de una hipótesis particular. Como cualquier otra regla ampliativa de desprendimiento, se reconoce que es falible; sin embargo, quienes la proponen sostienen que es, en términos generales, confiable. A continuación presentamos una representación familiar de

8 Josephson, John, "Symposium: Abductive Inference: On the Proof Dynamics of Inference to the Best Explanation", Cardozo Law Review, núm. 22, 2001, pp. 1621-1642. 
la regla de inferencia en cuestión, junto con algunos comentarios. 9

\section{Esquema General de la Inferencia a la Mejor Explicación}

1) e(1), e(2)... e(n) son los hechos prominentes a explicar

2) $h(1), h(2) \ldots h(n)$, cada una explica e(1), e(2),.. e(n).

Nótese que esta premisa constituye un alejamiento muy importante respecto de modelos de explicación anteriores (por ejemplo, el denominado modelo nomológico deductivo asociado con C. G. Hempel y muchos otros), los cuales exigían como condición necesaria para que $\mathrm{h}$ genuinamente explicara e, que fuera conocida la verdad de $h$ en forma independiente, o al menos que fuera altamente probable. Es decir, las teorias de la explicación anteriores suponian que ya habiamos establecido las credenciales epistémicas del explanas y que simplemente nos encontrábamos tratando de decidir si explicaba lo que pretendia explicar. Asimismo, las anteriores teorías de la explicación negaban que fuera coherente aseverar, como lo hace la IME, que diversas hipótesis incompatibles entre sí expliquen los mismos hechos. En contraste, la IME deja en el aire el status epistemológico de cada $h$ al momento de determinar si efectivamente las hipótesis en cuestión explican o no e(1), e(2),... e(n). La legitimación epistemológica de $h$, en todo caso, emerge solamente en virtud de haber determinado que esa $h$ es la mejor explicación de e(1), e(2), e(n) (como puede verse en 4).

9 Hill Lycan ha descrito un esquema de la IME en términos un poco más sintéticos, del modo que sigue:

$\mathrm{F}(1), \ldots \mathrm{F}(\mathrm{n})$ son los hechos.

La hipótesis H explica $\mathrm{F}(1), \ldots \mathrm{F}(\mathrm{n})$ ("explica" en este punto, debe entenderse en el sentido de "los explicaría de ser cierta)".

Ninguna otra hipótesis en competencia explica tan bien $\mathrm{F}(1)$ como lo hace $\mathrm{H}$.

Por tanto, (probablemente) $\mathrm{H}$ es verdadera.

Lycan, William, Judgment and Justification, núm. 129, 1988. 
3) Minuciosamente se han buscado explicaciones rivales de e(1), e(2)... e(n); sin embargo, el proceso de búsqueda sólo ha arrojado $h(1), h(2) \ldots h(n)$

Los proponentes de la IME explícitamente reconocen que el conjunto de hipótesis explicativas bajo consideración rara vez, si no es que nunca, serán tomadas como la unión exhaustiva de todas las hipótesis posibles que explicarian los hechos prominentes. Como un simpatizante del modelo, Kola Abimbola dice: "...la inferencia a la mejor explicación no requiere que esperemos a que todas las explicaciones posibles estén dentro del conjunto. Simplemente inferimos la mejor de las explicaciones disponibles... a no ser que estemos en camino de hallar otra buena explicación". 10

4) $h(1)$ es la mejor explicación en el conjunto (h(1), h(2),... $\mathrm{h}(\mathrm{n})$

El significado de "mejor" en este punto es un asunto sumamente controvertido entre los autores. Gil Harman ha dicho que "lo bueno" de una hipótesis explicativa depende de su simplicidad, su plausibilidad, y de la ausencia de elementos ad hoc en ella. Paul Thagard ha sugerido que una hipótesis califica como la mejor explicación sólo si es capaz de explicar diferentes clases de hechos, si posee simplicidad (entendida como una reflexión acerca de la naturaleza y el número de presupuestos que la hipótesis en cuestión requiere para explicar los hechos dados), si exhibe analogías apropiadas con otras hipótesis conocidamente exitosas, y si es fuertemente coherente con nuestras creencias de trasfondo. En su intento por abordar el problema de las virtudes explicativas de una hipótesis, John Josephson comenta que éstas tienen que ver con la consistencia interna de la hipótesis, con su poder predictivo, con qué tan decisivamente sobrepasa a sus rivales, y con la minuciosidad con que se buscaron hipótesis rivales. Bill Lycan incluye la sim-

10 Abimbola, Kola, "Abductive Reasoning in Law", Cardozo Law Review, núm. 22, pp.1683 at 1689 (las cursivas son nuestras). 
plicidad, el poder explicativo, su alto grado de comprobabilidad, y la coherencia con las creencias de trasfondo, como virtudes explicativas. ${ }^{11}$ Aunque podemos encontrar puntos de contacto, es obvio que no tenemos nada parecido a un consenso acerca de aquello que precisamente hace que una hipótesis sea mejor que otra(s). ${ }^{12}$ Antes de dejarnos deslumbrar por su resplandor, este aspecto en si mismo debería activar las alarmas respecto de si los procedimientos de la IME pueden ser identificados de un modo transparente, ya que si no nos es posible determinar claramente cuándo es mejor una hipótesis que otra, evidentemente no podemos decidir cuál de ellas es "la mejor". Pero dejaré esas preocupaciones de lado, toda vez que no son cruciales para mi argumento. ${ }^{13}$ Por mor del argumento simplemente asumiré que contamos con un mecanismo para determinar cuál de entre cualquier grupo de hipótesis rivales es la mejor.

5) Por tanto, $\mathrm{h}(1)$ es probablemente verdadera.

Ciertamente, todos los modelos de IME enfatizan que la mera existencia de un conjunto de hipótesis rivales que explican ciertos eventos, no garantiza que alguna de ellas siquiera califique como una "buena" explicación, mucho menos como "la mejor". Por ejemplo, si todas las explicaciones para un conjunto de hechos fueran ad hoc, entonces Harman negaria que alguna de ellas califique como la "mejor explicación”. Thagard diría lo mismo si ninguna de ellas fuera

11 Lycan, William, op. cit., nota 9, 130.

12 Otras propuestas familiares de virtudes explicativas incluyen la plausibilidad, la economía, la elegancia, la probabilidad, la responsabilidad epistémica (es decir, la búsqueda minuciosa de alternativas), la alta probabilidad posterior, la intuitividad, y la refutación de alternativas.

13 Desembrollar este particular lío (el cual podriamos denominar, siguiendo el epígrafe, "el problema de Schum"), implicaria un ensayo independiente. Simplemente dejaré asentado aquí que no existe unanimidad respecto de lo que son las virtudes explicativas y, en lo que resta del ensayo, supondré generosamente, por mor del argumento, que hay salida a este problema. Una postura menos indulgente instaría a los defensores de la IME, a volver a sus pizarrones hasta que estén en condiciones de contar una historia unificada acerca de en qué consisten las virtudes explicativas. 
simple, o tuviera la capacidad de explicar diferentes clases de hechos. Si no ha habido un minucioso proceso de búsqueda de alternativas, Josephson diría que ninguna de las hipótesis disponibles podría contar como la mejor explicación. Este punto es de crucial importancia, ya que tiene que ver con el status epistemológico que los autores de la IME desean conferir a una hipótesis en virtud de ser la mejor explicación. Como queda claro en (5), el modelo garantiza la suposición de que la mejor explicación de un conjunto de hechos, es probablemente verdadera. Lo anterior no gozaría de la menor plausibilidad si el modelo permitiera que esta regla de inferencia se aplicara en situaciones en las que todas las hipótesis disponibles fueran débiles o deficientes, o en las que el proceso de búsqueda de rivales haya sido menos que intenso y sistemático.

\section{ESTÁNDARES DE PRUEBA JURÍDICOS E IME}

En cualquier proceso penal, tenemos al menos dos historias o narrativas o conjuntos relacionados de aserciones, en juego: aquella ofrecida por el fiscal y la que ofrece el inculpado. Incluso cuando el inculpado no ofrece una historia como tal, sigue proponiendo una hipótesis, a saber, que la forma en que la otra parte da cuenta de los eventos es totalmente falsa. Toca al juez o al jurado el decidir no cuál de las historias o hipótesis es verdadera, sino si la parte sobre quien recae la carga de la prueba, ha establecido su historia al nivel de prueba requerido. Claro está que el acertijo es precisamente cómo debe hacer el juez o el jurado para realizar esa determinación. La respuesta familiar en los círculos anglosajones desde hace ya un par de siglos, ha sido que dicha determinación debe hacerse preguntando si el caso contra el inculpado ha sido probado, en lo que toca a la materia penal, mas allá de toda duda razonable (en adelante MATDR), o en materia civil, si la responsabilidad del demandado (por ejemplo en un asunto de daños y perjuicios) ha sido probada por una preponderancia de la evi- 
dencia. El problema, especialmente en el contexto penal, es que nadie está realmente seguro acerca de en qué consiste probar una hipótesis más allá de toda duda razonable. Particularmente en la época actual en que la Corte Suprema de los Estados Unidos ha decidido arrancar la noción del estándar penal de sus raíces tradicionales ubicadas en la teoría lockeana de la certeza moral, jueces y jurados por igual, muestran señales de una considerable confusión acerca de aquello en lo que consiste probar MATDR. ${ }^{14}$

Recientemente, algunos autores han sugerido que el modelo de la IME ofrece una solución satisfactoria a este enigma. Sostienen que probar la culpabilidad del acusado, más allá de toda duda razonable, es haber establecido que la historia del fiscal satisface los requerimientos asociados a una inferencia a la mejor explicación. Si no hay una clara determinación de que la narrativa de los eventos ofrecida por el fiscal es la mejor explicación de aquéllos, debe absolverse. Más específicamente, los autores en este campo han propuesto que una condición necesaria y suficiente (o necesaria pero no suficiente para algunos) para condenar al acusado, consiste en que la historia del caso propuesta por fiscal, califique como la mejor explicación.

Existen dos problemas distintos con la propuesta anterior. El primero es lo que Van Fraaseen, en otro contexto, ha dado en llamar "el problema de el mejor candidato de un conjunto malo". ${ }^{15}$ Imaginemos que tenemos dos hipótesis

14 Véase Laudan, Larry, "Is Reasonable Doubt Reasonable?", 9 Legal Theory, núm. 295, 2003, y Truth, Error and the Criminal Law: Essays in Legal Epistemology, Cambridge-Nueva York, Cambridge University Press, 2006.

15 En relación con el problema del mal conjunto, Van Fraaseen dice: "Creer algo es, por lo menos, considerar que es más probable que ese algo sea verdadero a que no lo sea. En este sentido, creer la mejor explicación requiere de algo más que una evaluación de la hipótesis en cuestión. Requiere de un paso adicional más allá del juicio comparativo consistente en que la hipótesis en cuestión es mejor que sus rivales actuales. Mientras que el juicio comparativo es, en efecto, una "ponderación (a la luz) de la evidencia", el paso adicional, llamémosle el paso ampliativo, no lo es. Para mi, tomar a la mejor hipótesis del conjunto X como más probable que su negación, requiere de una creencia previa de que es más probable, de antemano, encontrar la verdad en X a que no lo sea". Van Fraassen, Bas, Laws and Symmetry, núm. 143, 1989. 
en operación, h1 y h2, proferidas respectivamente por el fiscal y por el acusado. Supongamos también, que el jurado determina correctamente que $h 1$ es mejor que $h 2$, y por tanto, $h 1$ es, en este contexto, la mejor hipótesis ofrecida. ¿Justificaria esto una condena? Claramente no, al menos si no se agrega algo más. Si ninguna de las hipótesis acerca del caso es más o menos buena, entonces el jurado tiene la obligación de rechazar la hipótesis de culpabilidad y conceder una absolución, a pesar de que la explicación del fiscal haya sido la mejor. Esto es totalmente obvio y no requiere de mayor discusión. En principio, este problema puede disolverse demandando que el espacio de explicaciones posibles sea asiduamente configurado (como en el 3 de arriba).

El problema más intrigante no emerge cuando ambas partes cuentan con historias deficientes, sino cuando se ofrecen hipótesis razonablemente plausibles por ambas. Es así que a esto le podemos llamar el problema del mejor candidato dentro de un conjunto bueno. Supongamos que, de acuerdo con cualquier criterio concebible de lo que implica que una explicación sea buena, $h 1$ es superior a $h 2$. Se trata, en breve, de la mejor explicación aún sin calificarse como tal; sin embargo, si la $h 2$ del acusado no es una explicación particularmente mala, nuevamente se requeriría de una absolución. Lo que tenemos es una situación presumiblemente común en la que el modelo de la IME demandaría una condena (debido a que estamos suponiendo que no hay controversia en torno a que la historia del fiscal es la mejor explicación de los eventos del caso) a pesar de que todas nuestras intuiciones acerca de lo que significa MATDR nos indiquen que se requeriría de una absolución. En suma, la mejor explicación simplemente no es suficiente en el caso de un proceso penal.

Algunos teóricos de la IME aparentemente han entendido que el modelo necesita de alguna clase de suplemento en el caso de los procesos penales; sin embargo, no han formulado explícitamente los dilemas que el modelo enfrenta en los 
términos en que lo he hecho. Vale la pena echar un vistazo a la forma en que proponen lidiar con este embrollo. Paul Thagard, por ejemplo, ha dicho que las reglas convencionales de la coherencia y de la IME requieren ser suplementadas con una premisa adicional, a saber: "Desde la perspectiva de la teoría de la coherencia explicativa, la duda razonable puede verse como un constreñimiento adicional, el cual requiere que las hipótesis de culpabilidad sean sustancialmente más plausibles que las hipótesis de inocencia". ${ }^{16}$

La fuerza de este "constreñimiento adicional" radica en decir que la mejor explicación, como usualmente es entendida (y como es definida por el esquema 1-5), no es suficiente para que el fiscal obtenga la victoria; adicionalmente su teoría debe ser mucho, pero mucho mejor que la historia del acusado. Esta acertada observación por parte de Thagard es aplaudible, pero su efecto es alejar los vientos favorables de las velas del barco de la IME. Recordemos que una de las principales motivaciones para buscar en los modelos de la IME era, en primer lugar, remediar las deficiencias reconocidas en la noción actual de prueba más allá de toda duda razonable. Supuestamente la IME constituiría un rival de otros esfuerzos (como el de los teóricos de la probabilidad) por sustituir o, al menos, reformular el estándar MATDR. Pero si la aplicación de la IME a los procesos penales va a requerir que, de alguna forma, usemos precisamente la noción de MATDR para definir esa "laguna" de plausibilidad que debe haber entre la plausibilidad de la historia del fiscal y la del acusado, no es claro cómo la IME será menos ambigua que la propia noción de MATDR (a falta de una definición clara de lo que significa que la hipótesis de culpabilidad sea sustancialmente más plausible, la regla de Thagard parece invitar a confusiones e interpretaciones disparatadas del estándar ¿a caso Thagard realmente cree que los jurados, sin mayores problemas, podrian

16 Thagard, Paul, "Why wasn't O.J. Convicted? Emotional Coherence in Legal Inference”, Cognition and Emotion, núm. 17, 2003 pp. 361-366 y 367. 
frecuente o fácilmente alcanzar consenso acerca de si el caso del fiscal fue "sustancialmente más plausible" que el del acusado?).

En efecto, si el juez o el jurado tienen que resolver respecto de si la teoría del caso propuesta por el fiscal no es meramente más plausible que la del acusado, sino que la diferencia en sus respectivas plausibilidades es lo suficientemente grande como para satisfacer las demandas de MATDR, entonces no queda nada claro por qué deberian tomarse siquiera la molestia de descifrar las sofisticaciones del modelo de la inferencia a la mejor explicación. A menos de que la IME tenga algo específico que decir sobre lo que se prueba más allá de toda duda razonable, no podemos tratarla como un mecanismo para resolver lo que Allen y Pardo llaman el problema a nivel macro, consistente en explicitar la estructura del estándar de prueba. Es difícil ver cómo la IME se vuelve algo más que una rueda estática si su utilización en un proceso penal todavía depende de una noción de "prueba más allá de toda duda razonable", ajena a la propia IME.

Problemas de otro tipo afligen a un segundo intento, ampliamente citado, de usar la IME para capturar la lógica de un proceso penal, me refiero a la propuesta de Josephson, quien en un largo artículo dedicado a elogiar los méritos de la IME, define al estándar de prueba penal de la siguiente manera:

Esto sugiere una definición para el estándar judicial "más allá de toda duda razonable". La culpa de un sujeto ha sido establecida más allá de una duda razonable cuando no existe alguna explicación alterna plausible de los datos, que no implique la culpa del acusado. Una explicación es plausible si es internamente consistente con los hechos conocidos, no altamente inverosímil, y debe representar una "posibilidad real" no una mera posibilidad lógica. Una posibilidad real no supone violación alguna de las reglas de la naturaleza, ni tampoco supone algún comportamiento que sea completa- 
mente único y que no tenga precedentes, ni supone alguna cadena improbable de coincidencias. ${ }^{17}$

Aparentemente la idea es que una condena está justificada sólo si la teoría del caso ofrecida por el fiscal es plausible y no existe alguna teoría alterna plausible que sea compatible con la inocencia del acusado. Este principio, el cual fuera formulado por Ron Allen más o menos en términos idénticos hace una década, 18 no es un mal intento de formular el estándar de prueba penal. A diferencia del principio anterior de Thagard, éste no requiere de una noción independiente y autónoma de prueba MATDR, ni de una calibración de los grados relativos de plausibilidad de las hipótesis rivales. En lugar de ello, pretende reemplazar o explicar precisamente aquello que debe entenderse por MATDR; sin embargo, la pregunta clave para nosotros es simplemente ¿qué tiene que ver éste estándar de prueba con la IME?

La propuesta de Josephson es lo que podríamos llamar un modelo de inferencia a la única explicación plausible. Aquí no nos damos a la tarea de realizar ninguna de las actividades centrales de la IME; por ejemplo, no nos preguntamos si la teoría del fiscal es la mejor explicación acerca de

17 Josephson, John, Cardozo Law Review, at 1642. El tercer enunciado de la cita causa perplejidad. Leemos que "una explicación es plausible si es... (entre otras cosas) no altamente inverosimil...". Esta respuesta es tan informativa como aquella que dio un juez en un caso federal en el territorio de UTA, quien al ser cuestionado por el jurado sobre lo que era una duda razonable, respondió que "no era una duda no-razonable" (Dunbar v. US, 156 US 185 (1894). Esto no fue una respuesta, se trataba de una tautología. Pero iqué tal si el juez hubiera contestado, siguiendo las lineas de Josephson, que una duda razonable no era una duda altamente no-razonable? Josephson parece tolerar que una hipótesis medianamente inverosímil pueda considerarse, también, como plausible, y que sólo aquellas creencias disparatadamente inverosimiles, no pueden contar como hipótesis plausibles, en las circunstancias apropiadas.

18 Allen escribió: "Por su parte, esto significa que la prueba jurídica es de carácter comparativo; la cuestión trata de la plausibidad relativa de los casos de las partes; no tiene que ver con la probabilidad cardinal, concebida como una frecuencia relativa, o un estado subjetivo de creencia, de cierto estado de cosas. La analogía en materia penal es que el fiscal debe demostrar un caso plausible de culpabilidad, y que no haya un caso plausible de inocencia". Allen, Ronald, "Rationality, Algorithms and Juridical Proof: A Preliminary Inquiry”, 1 Int'l J. Evid. \& Proof, núm. 254, 1997. 
los hechos del caso. En su lugar, tratamos de determinar si se trata de la única explicación razonable de esos hechos, lo cual es un asunto diferente. Determinar si la historia del fiscal es mejor que sus rivales conocidas que a su vez también explican los hechos del caso, lo cual constituye la cuestión principal en el modelo de la IME, no es lo que importa. Ni siquiera se le puede encontrar en la periferia del análisis que Josephson hace acerca del estándar de prueba.

Es crucial recordar que el objetivo original de acudir al modelo de la IME era que éste nos instruyera sobre qué hacer cuando tenemos varias explicaciones rivales, prima facie plausibles de los mismos hechos sobre la mesa. Sin embargo, en este pasaje, Josephson nos está diciendo que una condena está justificada siempre y cuando haya sólo una explicación plausible de los hechos, la del fiscal. ${ }^{19}$ En este sentido, la definición que Josephson ofrece sobre MATDR implica el repudio de la regla de desprendimiento asociada al modelo de la IME. En concreto, Josephson nos insta a rechazar la mejor explicación de los eventos si hay una menos buena sobre la mesa, siempre y cuando esta última exhiba una plausibilidad mínima y haya sido propuesta por el acusado. Esta regla explicitamente demanda el rechazo de la mejor explicación, en el entendido de que esto se haga cuando hay otra que presenta al acusado como inocente. Irónicamente, es precisamente la aceptación de la mejor explicación para lo que el modelo de la IME fue diseñado. El objetivo que el proyecto de la IME ha querido lograr todo este tiempo es que estamos justificados en aceptar como verdadera a la mejor explicación de un conjunto de hechos. La versión de MATDR de Josephson nos pide rechazar la verdadera (de acuerdo con la certificación del mo-

19 Si la tomamos en su sentido literal, la definición de Josephson no requiere siquiera que la hipótesis de culpabilidad del fiscal sea plausible, mucho menos que sea la "mejor"; sólo requiere de la ausencia de una hipótesis plausible compatible con la inocencia "la culpa de alguien ha sido establecida más allá de toda duda razonable cuando no existe una explicación alterna plausible de los datos que no implique la culpabilidad del acusado"). Confio en que Josephson no intenta ser exacto aquí, ya que no puedo imaginar que trate de imponer tan débiles constreñimientos al fiscal. 
delo de la IME) teoría del caso, mientras haya una explicación menos plausible de los hechos que sea compatible con la inocencia del sujeto en cuestión. Repito, lo que sea que esté pasando aquí, no se trata de una inferencia a la mejor explicación.

Dicho lo anterior, un defensor de la IME podría responder:

Tenemos que entender simplemente que la "mejor" explicación en apariencia, no es necesariamente la mejor en un proceso penal particular. Lo que se requiere para que algo califique como la "mejor" explicación en un proceso penal es la ausencia total de una hipótesis plausible de inocencia. Eso es lo que "mejor explicación" significa en el contexto del derecho penal.

Dejemos de lado el hecho de que esta sugerencia rompe con el vínculo clave al interior del modelo de la IME, entre que una hipótesis sea la mejor explicación y que esa misma hipótesis sea, por tanto, probablemente verdadera (debido a que esta versión nos exige rechazar hipótesis que de otro modo serian certificadas como verdaderas por el modelo original de la IME). El aspecto más problemático de la propuesta es que implica la contextualización radical del modelo de la IME. Lo que en cierto contexto (digamos la ciencia o la historia) es la mejor explicación y, por tanto, lo que es razonable creer, no lo es más en otros contextos (como el derecho). Contextualizar así lo que cuenta como la "mejor" explicación atendiendo a los diferentes escenarios de toma de decisiones, priva al modelo de cualquier pretensión concebible de generalidad. Si la mejor explicación en un caso civil no es la mejor explicación en un caso penal, y si ninguna de aquellas necesariamente cuenta como la mejor explicación en investigaciones científicas o históricas, el resultado es que no puede sostenerse que la IME sea un mecanismo general para identificar creencias razonables o probables. Si el significado de "mejor" varía de un contexto a otro, una y la misma proposición puede ser la "mejor" en cierta situación y "menos que la mejor" en otra. Esto impli- 
caría, por ejemplo, que fuera razonable creer en un juicio civil, que O. J. Simpson asesinó a su esposa, pero que fuera irracional hacerlo en un proceso penal.

Sin embargo, el crítico sugeriría: ¿a caso no fue eso lo que pasó en el caso de Simpson? Esta pregunta confunde credibilidad y prueba. Lo que el modelo de la IME debe hacer, si se trata de una teoría general para certificar como racionales ciertas creencias (en efecto, eso es lo que pretende ser), es aseverar que, dadas la misma evidencia y las mismas creencias rivales, una que es creíble en una situación, lo será en otra. La razón por la que la IME no funciona como un estándar de prueba es porque aspira a ser un estándar universal de credibilidad y no una forma de medir el grado de prueba que sea sensible al contexto. La IME fracasa como estándar de prueba precisamente porque los estándares de prueba son locales y dependen del contexto específico. Éstos (los estándares de prueba) tienen que ver no con la credibilidad general, sino con el grado de fuerza probatoria que se demanda en una situación específica. Los modelos de la IME se restringen a la pregunta ¿qué es aquello que razonablemente puedo creer? Concediendo la importancia de la pregunta anterior, prácticamente en ningún caso se trata de la pregunta correcta, tratándose del contexto jurídico. La pregunta relevante (concerniente a la filosofía del derecho) es:¿qué debería aceptar como probado? Todos entendemos que los estándares de prueba son vehículos para la distribución de los errores, los cuales se informan de la determinación de los costos y beneficios asociados a dichos errores. Es debido a que esos costos y beneficios varian de un contexto a otro que usar un modelo que simplemente divida a las proposiciones en aquellas que son razonablemente creíbles y aquellas que no lo son (lo cual constituye el objetivo del modelo de la IME), indiferente a los costos de los errores previsibles, es como tratar de clavar un clavo usando un desarmador. Simplemente es la herramienta errónea para el trabajo. 
Sugerí en algún momento que podemos denominar al de Josephson como el modelo de la única explicación plausible; sin embargo, esa descripción es adecuada sólo en el caso de las condenas. En el caso de las absoluciones, podriamos llamarlo el modelo del rechazo de la mejor explicación, ya que sin duda alguna (y quizás en forma correcta), Josephson piensa que un acusado puede ser absuelto aún cuando su explicación de los eventos sea claramente inferior a la explicación proporcionada por el fiscal. ${ }^{20}$

Como hemos observado, al ser enfrentados a la cruda realidad del estándar de prueba penal, tanto Thagard como Josephson se encuentran obligados a admitir que frecuentemente es adecuado rechazar la mejor explicación de los hechos en juicio. Esto no permite escapatoria a la conclusión de que un modelo cuyo objetivo consiste en caracterizar cuándo debemos aceptar una hipótesis en términos de sus virtudes explicativas específicas, nos ofrece muy poca instrucción acerca de las condiciones que deben ser satisfechas por el fiscal en un proceso penal. En resumen, el esquema de la IME fracasa en tanto que no puede modelar la inferencia medular que conduce a concluir la culpabilidad o la inocencia. Sirve sólo cuando se introducen constreñimientos periféricos que contradicen la propia regla de desprendimiento que la IME exige específicamente.

¿Podría la IME tener mejores resultados dando cuenta del estándar de prueba civil? Podría parecer así, ya que al juez o jurado en un juicio civil se le pide determinar cuál de las teorias ofrecidas por las partes respecto de los hechos es más probable o más convincente. Esto nos suena a: ¿quién tiene la mejor, o al menos la mejor explicación? Pero las apariencias suelen ser engañosas. Recordemos que la regla de la IME no garantiza automáticamente el desprendimiento de las más fuerte de dos hipótesis rivales, lo cual es

20 Josephson podría responder argumentando que los principios de la IME tienen que ser usados para determinar si la historia del acusado es o no plausible; sin embargo, notemos que la definición de Josephson de MATDR ya se encuentra especificando las condiciones de plausibilidad mínima sin referencia alguna a una determinación de que la explicación del acusado tenga que ser la "mejor". 
lo que necesitamos si deseamos capturar la noción del estándar civil. Al contrario, la IME insiste, en forma correcta, para procesos de toma de decisiones no jurídicos, que ninguna hipótesis del conjunto inicial puede desprenderse a menos que exhiba mayores elementos para ser la mejor explicación que sus conocidas rivales. La IME establece las condiciones mínimas que deben ser satisfechas por cualquier hipótesis que legítimamente se desprenda como "la mejor explicación" (esta es la razón de ser del punto 4 del esquema de la IME).

Como hemos visto, una explicación certificada por la IME debe exhibir un alto grado de coherencia con otras creencias de trasfondo, debe ser internamente consistente, debe explicar diferentes clases de hechos, debe ser simple, no ser ad hoc, etcétera. Cuando faltan estas últimas virtudes, la IME no certifica ninguna inferencia. Sin embargo, los juicios civiles no son así. Los jueces o jurados no pueden decir: "Aunque la historia del actor es más fuerte que la del demandado, no dictaré sentencia alguna, dado que ninguna de las partes tiene una historia lo suficientemente buena que contar". Según las reglas actuales, si el actor tiene una mejor historia que la del demandado, debe ganar el juicio, aun cuando su teoría del caso fracase en satisfacer los requerimientos a efecto de calificarla como la mejor explicación. ${ }^{21}$ En este estado de cosas, la IME es simplemente demasiado demandante como estándar de prueba civil. Para aplicar el modelo al derecho civil, como mínimo, tendríamos que desechar la condición 4) de nuestro esquema, pero dificilmente eso sería adecuado, ya que un modelo de inferencia a la mejor explicación que no requiriera que la explicación desprendida satisfaga la demanda de ser "la mejor" claramente es un oxímoron.

21 Uno podría imaginar un sistema de adjudicación para los juicios civiles en que el juez o el jurado automáticamente fallaran a favor del demandado a menos que la teoría del caso propuesta por el actor fuera, en efecto, la "mejor explicación" en el sentido en que la IME emplea el término. Pero esa sería una práctica diferente a la que actualmente prevalece en el derecho civil anglosajón. 
Quienes proponen la IME enfrentan otras dificultades en su intento por capturar la lógica de los procesos judiciales. Sin entrar en detalles, mencionaré dos de ellas: tanto en el derecho civil como en el penal, se debe lidiar con la carga de la prueba (la cual, en ocasiones pasa de una parte a otra). Tenemos que preguntarnos ¿de qué manera puede un modelo como el de la IME, que no toma en consideración la participación de las partes en los procesos de investigación, capturar la idea de que las partes que fracasen en cumplir con su carga de la prueba perderán, sin importar que hayan proferido la "mejor explicación" de los hechos del caso? Por otra parte, tenemos también los diversos principios de distribución de los errores que están en la base de un proceso penal: el acusado tiene derecho al beneficio de la duda (in dubio pro reo) en un caso límite; al inicio de un proceso penal, el acusado tiene la presunción de inocencia, la cual debe ser derrotada para garantizar una condena; las reglas que excluyen evidencia limitan drásticamente el rango de evidencia relevante que puede ser admitida en un proceso. Ninguna de las prácticas mencionadas parece embonar en forma natural, en el esquema de la inferencia a la mejor explicación.

\section{CONCLUSIÓN}

Hemos podido observar situaciones en las que: a) ser la "mejor explicación" simplemente no es suficiente para garantizar el desprendimiento de una hipótesis (en el derecho penal); b) insistir en la "mejor" explicación es demasiado demandante (en el derecho civil); y c) la "mejor" no es la mejor explicación posible, sino simplemente la mejor de las disponibles. Nadie ha mostrado cómo derivar un estándar de prueba tipo MATDR del esquema inferencial de la mejor explicación. Esto se debe a que una hipótesis puede ser la mejor explicación conocida de los hechos, incluso luego de una concienzuda búsqueda de alternativas, sin que ello la certifique como verdadera más allá de toda duda razonable. 
Puede ser que sea "probablemente verdadera" (lo cual es lo más que el esquema de la IME permite inferir) sin que ello implique que sea lo suficientemente creíble como para remover toda duda razonable. Peor aún, la estructura de un proceso penal requiere, bajo una variedad de circunstancias diferentes, que se llegue a conclusiones contrarias al esquema inferencial que se encuentra en el núcleo de la IME. Por ejemplo, si el acusado proporciona una historia exculpatoria aunque sea medianamente plausible acerca de los eventos asociados con el delito, debe ser absuelto, incluso si la teoría del caso propuesta por el fiscal indiscutiblemente ofrece la mejor explicación de la evidencia presentada durante el juicio. El punto simplemente es que los requerimientos de la IME son más débiles que los del estándar MATDR en cuanto a lo que el Estado debe probar. Este problema puede evitarse sólo si adherimos al modelo de la IME ciertos constreñimientos periféricos (por ejemplo, "la mejor explicación en un proceso penal ocurre sólo cuando el acusado no tiene una explicación plausible") que destruyen su intuición toral de que la genuina mejor explicación es la explicación que hay que considerar como verdadera. ${ }^{22}$

Distintas razones nos proporcionan bases para sospechar que la IME no hará mejor las cosas como un sustituto del estándar de prueba por una preponderancia de la evidencia, ya que el actor en un juicio civil puede asegurar un veredicto favorable, aun cuando su hipótesis no sea la "mejor" explicación.

Me apresuro a añadir que nada de lo que he dicho aquí debe tomarse como que estoy negando que ambas partes en

22 En un sentido distinto, los requerimientos de la IME son demasiado fuertes a efecto de capturar algunas de las circunstancias en las que procedería una condena. Por ejemplo, si el acusado no cuenta con ninguna historia plausible, y la que ofrece el fiscal es bastante plausible y bien soportada en la evidencia presentada en juicio, en ese caso, procedería una condena, aunque la hipótesis del fiscal no exhibiera algunos de los elementos de mejor explicatividad que los modelos de IME familiares tratan como pre-requisitos de una mejor explicación genuina (por ejemplo, la alta coherencia con creencias de trasfondo, la habilidad para explicar diferentes clases de hechos, la simplicidad, y la ausencia de elementos ad hoc). 
un juicio efectivamente tratan de explicar los hechos del caso. Mi argumento tampoco niega que los jueces o jurados generalmente valoran las historias desde una perspectiva holística, en lugar de hacerlo considerando los elementos individuales del delito aisladamente. Por otra parte, nada de lo dicho debe tomarse en el sentido de que esté implicando que las probabilidades subjetivas sean un camino más prometedor a efecto de comprender la prueba en el derecho. Lo único que se ha sostenido es que la IME es un modelo (quizás malo, quizás bueno, o incluso, insuficiente) acerca de lo que generalmente es racional creer, y no un modelo de la prueba jurídica. 